\title{
BMJ Open Estimate of global human papillomavirus vaccination coverage: analysis of country-level indicators
}

\author{
Jacqueline Spayne (D) ,,2 Therese Hesketh ${ }^{2,3}$
}

To cite: Spayne J, Hesketh T. Estimate of global human papillomavirus vaccination coverage: analysis of countrylevel indicators. BMJ Open 2021;11:e052016. doi:10.1136/ bmjopen-2021-052016

- Prepublication history and additional supplemental material for this paper are available online. To view these files, please visit the journal online (http://dx.doi.org/10.1136/ bmjopen-2021-052016).

Received 02 April 2021 Accepted 23 August 2021

\section{Check for updates}

(c) Author(s) (or their employer(s)) 2021. Re-use permitted under CC BY-NC. No commercial re-use. See rights and permissions. Published by BMJ.

${ }^{1}$ Department of Radiation Oncology, University of Toronto, Toronto, Ontario, Canada ${ }^{2}$ Institute for Global Health, University College London, London, UK

${ }^{3}$ Centre for Global Health, Zhejiang University School of Medicine, Hangzhou, People's Republic of China

Correspondence to Professor Therese Hesketh; t.hesketh@ucl.ac.uk

\section{ABSTRACT}

Background Mortality rates from cervical cancer demonstrate deep inequality in health between richer and poorer populations. Over 310000 women died of this preventable disease in 2018, mostly in low-income and middle-income countries (LMICs) where screening and treatment are beyond the capacity of health systems. Immunisation against human papillomavirus (HPV) offers a primary prevention strategy, but rates of vaccination uptake are unclear. Understanding coverage levels and factors affecting uptake can inform immunisation strategies.

Objectives The aim of this study is to evaluate the status of HPV vaccination coverage from nationally reported indicators and to estimate global coverage in a single year cohort of vaccine-eligible girls.

Design This study provides quantitative population-level estimates of important global health indicators. Using data from the Global Cancer Observatory and WHO/ UNICEF, incidence of and mortality from cervical cancer and HPV vaccination coverage are described for countries, categorised by income group. Characteristics of LMICs achieving high coverage are explored using selected development indicators from World Bank sources. Global HPV immunisation coverage is calculated and its impact on cervical cancer mortality estimated.

Results Incidence and mortality for cervical cancer correlate with poverty. Whilst all WHO member states report high infant measles vaccination rates, fewer than half report on HPV vaccination. Even amongst high-income countries, coverage varies widely. In upper-middle-income countries, there is a trend for higher coverage with increased health spending per capita. Four LMICs report good coverage levels, all associated with external funding. Global HPV immunisation coverage for 2018 is estimated at $12.2 \%$. Of the global cohort of 61 million 15 -year-old girls in 2018, 7000 are likely to die from cervical cancer, almost all in LMICs.

Conclusions Countries in all income groups must devise strategies to achieve and maintain higher levels of HPV immunisation. For all but the richest, affordability remains a barrier.

\section{INTRODUCTION}

The burden of cervical cancer

Over 310000 women died of this preventable disease in $2018,90 \%$ of whom were in

\section{Strengths and limitations of this study}

This study collates immunisation data submitted by all 195 WHO members for 2018.

- Data for human papillomavirus vaccination coverage are missing for over half of WHO members for 2018, limiting the conclusions which can be drawn from analysis.

- Data for immunisation coverage, cancer incidence and mortality and development indicators are drawn from three databases maintained by separate organisations for different purposes, potentially limiting comparability.

- Analysis is limited by variable methods used for data collection by individual countries including administrative, survey and estimated data.

- The study analyses a single year of reported immunisation data with the attendant risk that this might not be truly representative.

low-income and middle-income countries (LMICs). ${ }^{1}$

Cervical cancer is a sexually transmitted disease caused by the human papillomavirus (HPV). ${ }^{2}$ In 2018, there were an estimated 570000 new cases of cervical cancer worldwide. ${ }^{1}$ High-income countries (HICs) report age-standardised incidence rates (ASIRs) of 5-10 per 100000 women, with agestandardised mortality rates (ASMRs) as low as 2 per 100000 women. ${ }^{3}$ This compares to an ASIR over 40 per 100000 in much of subSaharan Africa, where ASMRs are 30-60 per $100000 .^{3}$

In HICs, the incidence of cervical cancer declined by half in the past 30 years largely due to screening ${ }^{4}$; the case fatality rate also declined as a result of treatment advances which can now achieve survival rates of up to $87 \%$ in early-stage disease ${ }^{5}$ and $70 \%$ with locally advanced disease ${ }^{6}$ In contrast, incidence in sub-Saharan Africa has increased over this period, with high case fatality rates ${ }^{7}$ due to late stage presentation ${ }^{8}$ and limited treatment availability. ${ }^{59}$ 


\section{Prevention of cervical cancer}

The Pap smear has enabled effective prevention of cervical cancer since the 1950 s. ${ }^{10}$ Alternative screening approaches requiring less skill and fewer resources include visual inspection with acetic acid ${ }^{11}$ and self-sampling for HPV DNA testing. ${ }^{12}$ All these methods require colposcopic follow-up to ablate abnormal epithelial cells.

The discovery of HPV as the causative agent of cervical cancer $^{2}$ offered the opportunity to develop primary prevention approaches. Early clinical trials of vaccines against HPV-16 and HPV-18, the strains responsible for $70 \%$ of cervical cancers, showed high efficacy against infection in adolescent girls. ${ }^{13}$ Additional strains (HPV-6 and HPV-11) are now included in a quadrivalent vaccine which offers effective prevention of precancerous cervical lesions, as well as precursors of vaginal, vulval, anal canal, penile and oropharyngeal cancers. ${ }^{1415}$ Population-based studies (all in HICs) demonstrated that, with greater than $50 \%$ vaccine uptake, HPV-16 and HPV-18 infections decreased by $68 \%$ in girls aged $13-19 .{ }^{16}$ Cross-protection against other HPV strains and infections in older women were noted, suggesting herd effects. ${ }^{17} 18$

HPV immunisation programmes began in 2007, mostly in HICs, ${ }^{19}$ and cost-effectiveness studies have shown clear benefits. ${ }^{20}$ Programmes have increasingly used schoolsbased implementation strategies supplemented by opportunistic delivery through primary care providers. ${ }^{21}$ Resource-stratified guidelines have been developed ${ }^{22}$ to encourage adoption of HPV immunisation programmes in LMICs, but barriers to implementation remain. ${ }^{23}$

The success of programme implementation remains unclear. A 2016 pooled analysis of data from published literature, government websites and donor aid sources estimated that only $1.2 \%$ of women had received a complete course of vaccine with wide variations: $33.6 \%$ of females aged 10-20years in developed regions, but only $2.7 \%$ in less developed regions. ${ }^{24}$ A review of 28 studies to 2013 also reported wide variations in coverage. ${ }^{25} \mathrm{~A}$ survey of 45 LMICs found high uptake could be achieved in school-based programmes ${ }^{26}$ but, where school enrolment was low, supplementary approaches were needed. ${ }^{27}$ Two recent reviews found wide differences in policy and uptake across Europe. ${ }^{28} 29$ Many HICs have registries providing estimates for coverage (see literature review in online supplemental file 1), but few such sources are available in LMICs. Furthermore, estimates based on widely different methodology cannot easily be compared across time or geography. A recent WHO/UNICEF report estimated that less than one in three girls live in a country with HPV vaccine in the immunisation schedule ${ }^{30}$ and, even where provided, many girls are not reached, regardless of country income levels. ${ }^{31}$

\section{WHO strategy towards the elimination of cervical cancer}

The key challenge for HPV immunisation is how to extend its benefits to most of the world's population. The WHO Global strategy for elimination of cervical cancer has adopted targets of $90 \%$ vaccination coverage, $70 \%$ twice-lifetime screening and $90 \%$ treatment of preinvasive lesions and invasive cancer by $2030 .^{32}$

Any evaluation of progress towards achieving these targets requires country-level statistics using reliable, consistent and clearly defined indicators. A database compiled by WHO and UNICEF, released in 2019, provides indicators of HPV vaccination coverage, as reported by member countries. ${ }^{30}$ The aim of this study is to provide a baseline estimate of global HPV immunisation coverage from these data, and to compare coverage levels between and among countries in different income strata.

\section{METHODS}

This study uses data from three publicly available databases to examine the burden of cervical cancer globally and evaluate coverage with HPV vaccination. Data are gathered at nation state level and analysed by strata of gross national income (GNI per capita), according to World Bank criteria. ${ }^{33}$ Categories are: HICs, uppermiddle-income countries (UMICs), lower-middle-income countries (here abbreviated as LrMICs, to distinguish from LMICs) and low-income countries (LICs).

\section{Global Cancer Observatory}

The global cancer observatory $(\mathrm{GCO})^{34}$ is an interactive web-based platform provided by the International Agency for Research on Cancer (the specialised WHO cancer agency). This includes estimates of incidence, mortality and prevalence of the main cancer types, by sex and age group. ${ }^{35}$ The GCO Today database ${ }^{34}$ was interrogated for country-level data for cervical cancer incidence and mortality in 2018, including absolute numbers and crude and age standardised rates per 100000 women.

\section{World Bank}

Classification of countries according to World Bank Income Groups was obtained for the 2018 calendar year. ${ }^{33}$ Demographic and economic data for 2018 were extracted from the World Bank Open Data web page. ${ }^{36}$ Mortality and school enrolment data were sourced from the World Bank Development Indicators database. ${ }^{37}$

\section{UNICEF-WHO immunisation databases}

Global immunisation data are requested annually by UNICEF/WHO from the Ministries of Health of every WHO member state ${ }^{38}$ and are collated and made available through the UNICEF website. ${ }^{39}$ The dataset 'WHO estimates of HPV immunisation coverage 2010-2018, ${ }^{40}$ (a copy is provided in online supplemental file 2) contains country-level first and last dose reported HPV vaccination coverage according to a locally defined age schedule and/or by age 15 .

To provide a comparator for HPV immunisation reporting, the UNICEF measles immunisation dataset was accessed ${ }^{39}$ to obtain 2018 indicators for percentage 
of infants receiving a first and second dose of measlescontaining vaccine.

The selected data from each of the three sources were downloaded and amalgamated into an Excel spreadsheet, matching countries to the $195 \mathrm{WHO}$ member list grouped by income strata.

\section{Cervical cancer incidence and mortality}

Cervical cancer incidence and mortality data were tabulated by country; incidence of and mortality from all other cancers (ie, all cancers excluding cervical cancer) was calculated by subtracting cervical cancer from all cancer. Data were expressed as crude incidence rates per 100000 females for age bands 0-24, 25-39, 40-54, 55-69 and over 70 .

\section{Immunisation coverage and development indicators}

Data for measles and HPV immunisation coverage and selected economic and development indicators were tabulated at country level. Scatter plots were used to demonstrate potential associations between these variables and HPV immunisation completion in 2018. Trend lines were added using Excel tools (using a least squares method).

\section{Estimation of global HPV immunisation coverage}

To estimate global HPV vaccination coverage, cohort size for each country was approximated from the following indicators provided by the World Bank for $2018^{36}$ :

a. Total female population age 5-14.

b. Female deaths age 5-14, using the formula:

$$
\text { Cohort size }=(a \div 10)-(b \div 2) \text {. }
$$

This provided an estimate of the female population cohort at age 15 . The numbers of vaccinated and unvaccinated girls in each national cohort were calculated. For non-reporting countries, immunisation rates were assumed to be zero. Estimates of predicted cervical cancer deaths in the unvaccinated cohort were calculated using the country-specific remaining lifetime mortality risk at age 15 , available from the GCO database. ${ }^{34}$ As the HPV vaccine is estimated to prevent infection causing $90 \%$ of cervical cancers, ${ }^{41}$ in the vaccinated cohort, the diseasespecific mortality rate was estimated at $10 \%$ of the unvaccinated cohort.

\section{Patients and public involvement}

Neither patients nor the public were involved in this study.

\section{RESULTS}

Demographic and cancer data, immunisation rates and selected development indicators are shown at country level in tables A-D in online supplemental file 3. Countries are categorised by income group, within which they are listed alphabetically. Data are given for 2018 or the most recent year available. Data sources are described in the Methods section.

\section{Burden of cervical cancer}

Estimates of ASIR and ASMRs in 2018 for cervical cancer are shown at individual country level in tables A-D in online supplemental file 3 . These data are summarised for income groups in figure 1 , stratified by age bands and compared with all other cancers. Cervical cancer is more common in younger age groups compared with other cancers and, in every age band, the rate of new cases of and deaths from cervical cancer is clearly associated with poverty.

\section{Immunisation reporting}

Immunisation reporting for 2018 submitted by individual countries to WHO is shown in tables A-D in online supplemental file 3. While all 195 participating countries submitted data on measles vaccination, only 78 countries reported on HPV immunisation programmes, of which two (Zimbabwe and Thailand) had delivered only first doses. Seventy-six countries reported on completed courses to the locally defined target female population. These data are included in tables A to D in online supplemental file 3, except where higher coverage was reported for completed courses by age 15 , suggesting vaccination catch-up. The HPV vaccine coverage data, therefore, provide a best-case scenario snapshot of vaccination coverage in 2018 for the 76 reporting countries.

Immunisation reporting by income group is summarised in table 1, showing the number of countries in each income group that submitted records of measles and HPV vaccination. Measles first dose vaccination coverage was over $80 \%$ in all but 35 countries, many of which were LrMICs or LICs affected by conflict (eg, South Sudan, Syria and Somalia). However, reporting of second dose measles vaccination showed lower coverage levels, particularly in LrMICs and LICs.

Similarly, the proportion of countries reporting HPV immunisation was related to income level although, even in HICs, there were significant gaps in reporting. To examine factors that might contribute to establishment and success of HPV immunisation programmes, selected development indicators were compared with rates of HPV completion.

\section{HICs and UMICs}

Scatter plots in figure 2 show 2018 HPV immunisation completion in HICs, plotted against four indicators: GNI per capita, health expenditure per capita, percent of health expenditure from private sources and female secondary school enrolment.

Data on HPV immunisation completion were available from 41 of 59 HICs. Of reporting nations, 11 recorded immunisation completion rates above $80 \%$ and only four had rates below $20 \%$. The charts in figure 2 suggest no correlations between the development indicators examined and levels of HPV immunisation in HICs.

The same set of indicators, plotted against HPV immunisation completion rates for UMICs, are shown in figure 3. 

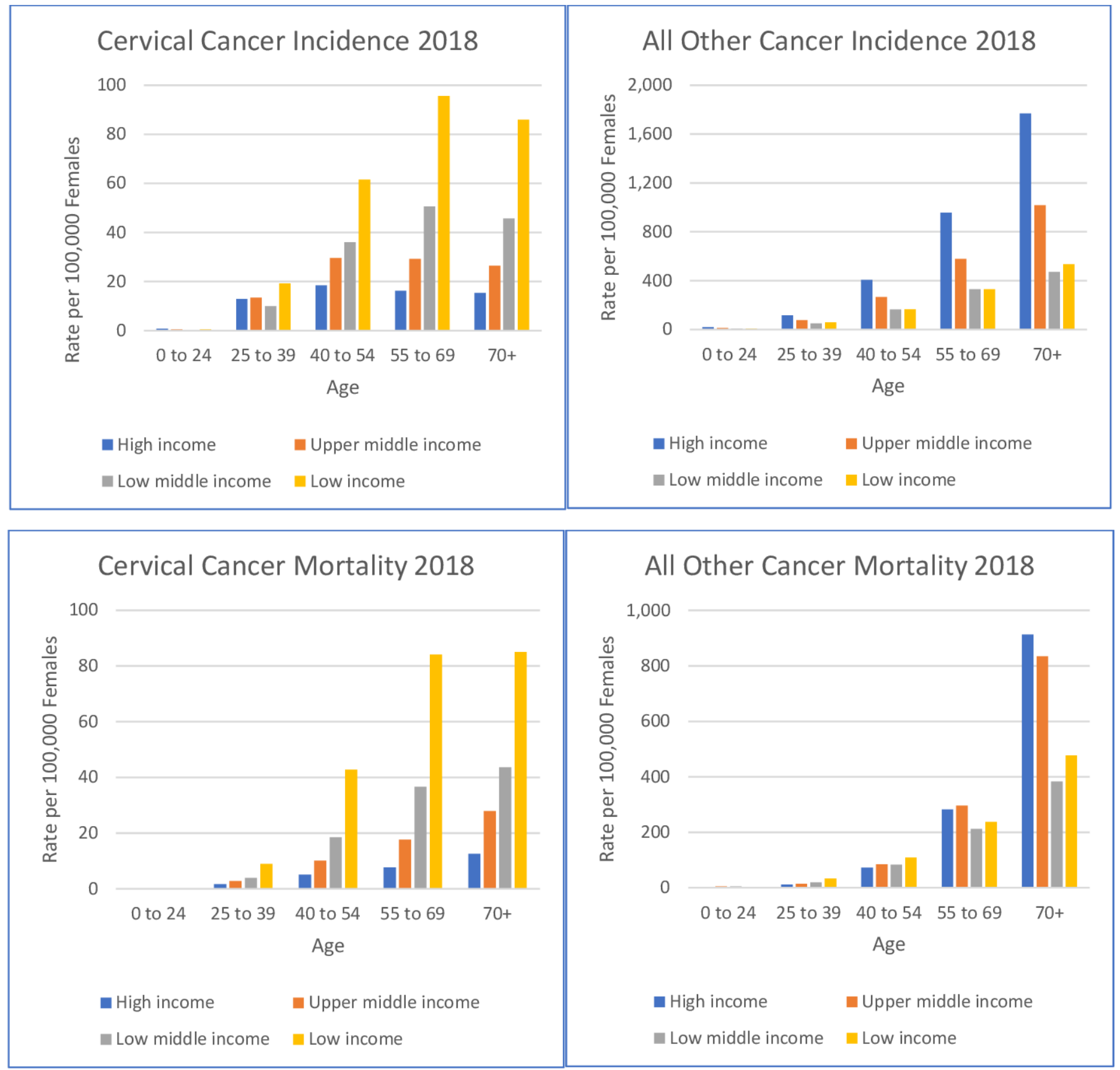

Figure 1 Incidence and mortality of cervical and all other cancers in 2018, by income group and age bands. Data are shown as crude rates per 100000 females. Note differences in scale of $\mathrm{X}$-axes.

Among 59 UMICs, 33 did not report on HPV immunisation; of the 26 that did, 7 had completion rates below $20 \%$ and only 6 achieved completion rates above $80 \%$. Of the development indicators analysed, there was no apparent correlation in UMICs between HPV immunisation completion rates and either the proportion of health expenditure from private sources, or the rate of female secondary school enrolment. The data, however, suggest trends relating both GNI/capita and health expenditure/capita (both at purchasing power parity, PPP) with HPV immunisation completion rates.

\section{LrMICs and LICs}

The World Bank income group classification for 2018 lists 46 LrMICs and 31 LICs among WHO members. Both groups have very few nations reporting on HPV immunisation completion (see tables C, D in online supplemental file 3). Of those that do, several are very small (eg, Bhutan, Micronesia and Sao Tome) and will not be discussed further here.

Two countries in each of the LrMIC and LIC groups report relatively high levels of HPV immunisation completion: Bolivia and Honduras (both at 61\%) among LrMICs; and Rwanda (at 84\%) and Uganda (at 72\%) among LICs. 


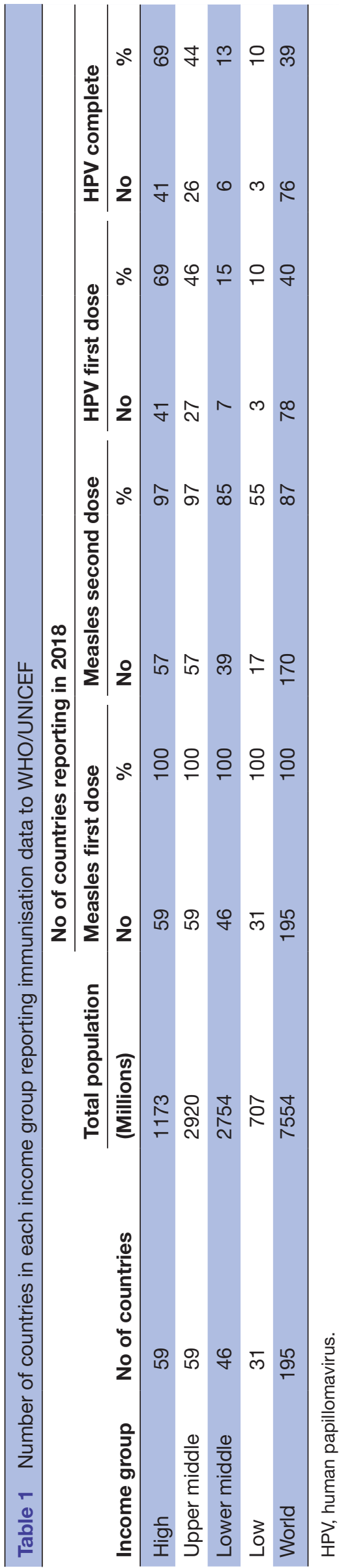

Estimate of global HPV immunisation coverage and preventable mortality

Country-level HPV immunisation rates and an estimated cohort size for 15-year-old girls in 2018 were used to calculate numbers of immunised and unimmunised girls in this single global cohort. These data are summarised for each income group in table 2.

If the absence of reporting indicates a lack of immunisation (which is far from clear), then the rate of HPV immunisation globally in this cohort was only $12.2 \%$. Extrapolating from this, approximately 7000 girls in this 1-year global birth cohort might be expected to die from cervical cancer, almost all in poorer nations.

\section{DISCUSSION}

This study shows that poverty is a major contributor to the burden from cervical cancer. Women die now from this preventable disease due to lack of knowledge, insufficient screening and an absence of diagnostic and treatment services. The data demonstrate that cervical cancer affects a younger age group than most cancers and that, at all ages, incidence and mortality from cervical cancer increase with poverty. Widespread HPV immunisation could resolve this inequity.

An important limitation of this study reflects the limitations of the data sources on which it is based. Estimates of cancer incidence and mortality in most HICs are based on registry data. In poorer countries such estimates may be less reliable as registries are often absent, incomplete or out of date. Cancer data are often based on household surveys and extrapolation from limited hospital data and neighbouring communities. ${ }^{42}$ It is likely that many cancer cases in LMICs go unrecorded. If so, then the gaps described in cervical cancer mortality between richer and poorer nations may be wider than estimated.

Similar criticisms apply to data for vaccination coverage. All WHO member states reported on infant immunisation in 2018 (shown here by $100 \%$ reporting of first measles vaccination), confirming the commitment and administrative capacity (at least in theory) to comply with reporting. However, fewer than half submitted data on HPV immunisation. Even in rich countries, these data are suspect because many do not have national organised programmes and/or do not maintain adequate registries (see literature review in online supplemental file 1). Coverage estimates based on survey data can be subject to selection and recall biases and may not differentiate between initiation and completion of vaccination courses. Where survey data are described in the literature for such countries, it suggests low rates of coverage,${ }^{43-46}$ associated with opportunistic approaches to HPV immunisation.

Unsurprisingly, economic and development-related indicators do not explain wide variations in coverage rates in HICs. Most have well-organised and well-funded public health programmes, exemplified by high levels of completed measles immunisation. Similarly, female secondary school enrolment is universally high, enabling 

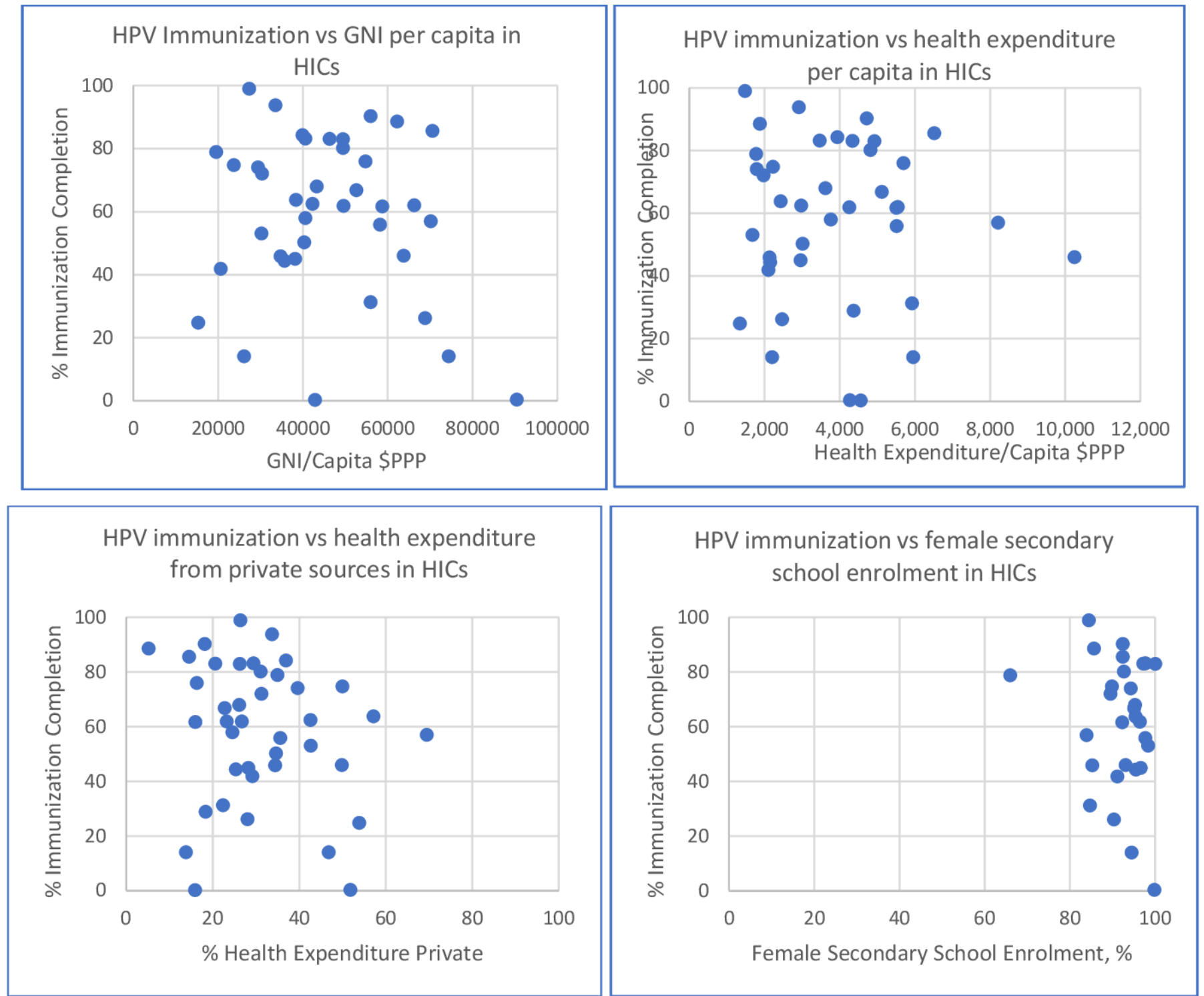

Figure 2 Scatter plots of development indicators against HPV immunisation completion in high-income countries (HICs) in 2018. GNI, gross national income; HPV, human papillomavirus; PPP, purchasing power parity.

organised HPV immunisation. Vaccine hesitancy is a barrier ${ }^{4344}$ although information campaigns can be effective to reverse falls in coverage. ${ }^{47}$

Just under half of UMICs reported on HPV immunisation coverage, but few achieved rates above $80 \%$. Most of these countries have organised health systems with excellent performance on childhood immunisation. They also have a high proportion of females enrolled in secondary school. It appears that, for countries in this wealth bracket, a critical factor may be total health expenditure per capita. It is notable that, while most HICs spent over US $\$ 1800$ /capita at PPP on health in 2018, only 2 of 59 UMICs reached this level and most were well below. While extensive missing data preclude tests for correlation, these findings suggest that, even for these relatively affluent countries, affordability remains a barrier for HPV, but not measles vaccine; it is notable that the cost of one dose of quadrivalent HPV vaccine to UMICs is estimated at around US\$15 compared with US\$0.69 for one dose of measles vaccine. ${ }^{48}$

Among the 46 countries in the LrMIC group, only half achieve second dose measles vaccine administration above $80 \%$; only 5 countries reported female secondary school enrolment above $80 \%$. It is, therefore, unsurprising that establishment of HPV immunisation does not appear to be a priority in these communities. However, there are two exceptions: Bolivia and Honduras, both with coverage rates of $61 \%$. Development indicators in these countries are mixed: while Honduras does well with second-dose measles, Bolivia does not; the opposite is true of female secondary school enrolment. Both are above the median in their income group for GNI/capita and for health spending per capita, though neither reaches the 90th percentile for either indicator. These indicators do not, therefore, explain the observed outperformance for HPV immunisation rates. Although neither country 

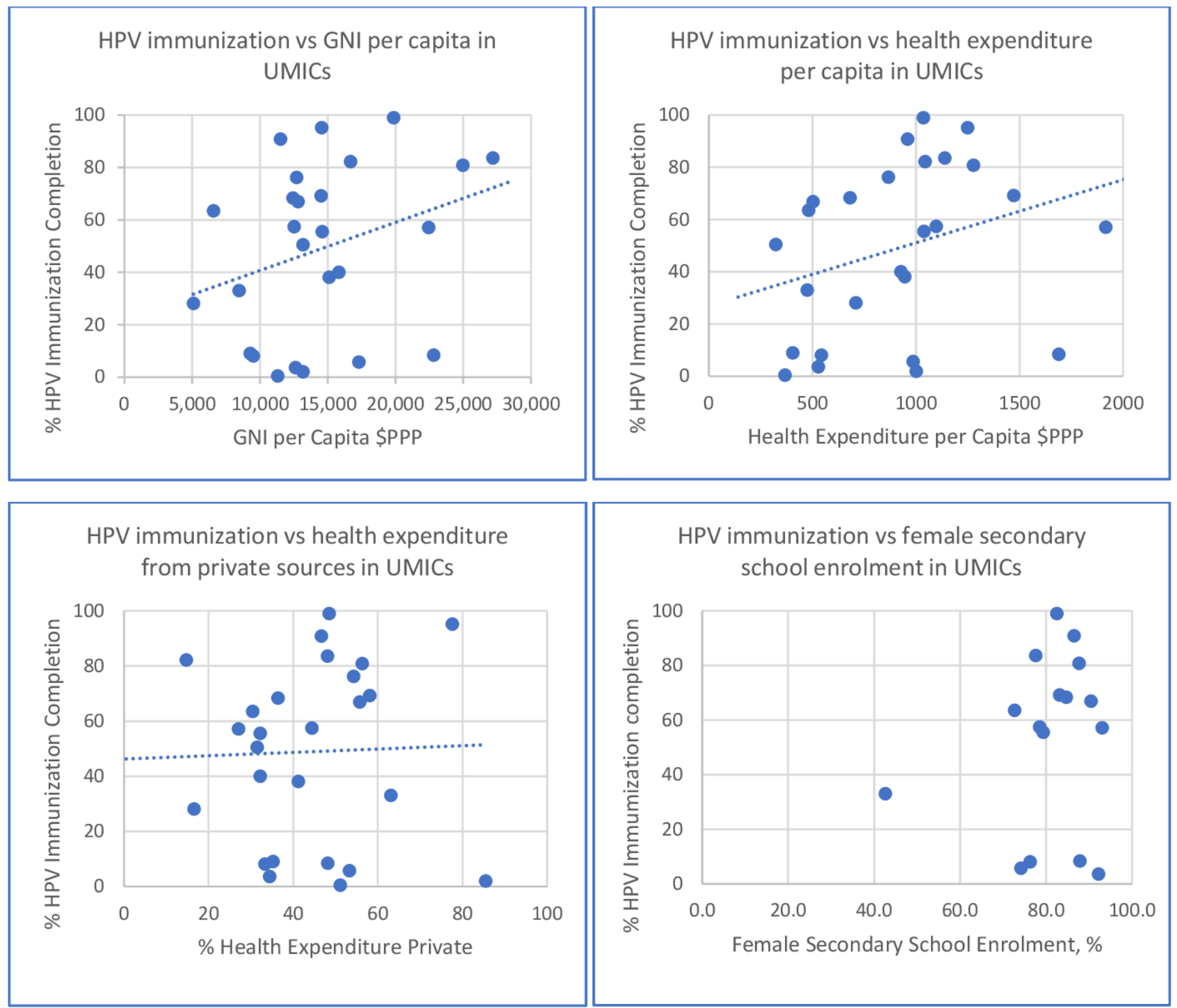

Figure 3 Scatter plots of development indicators against HPV immunisation completion in upper-middle-income countries (UMICs) in 2018. GNI, gross national income; HPV, human papillomavirus; PPP, purchasing power parity.

receives a significant proportion of health funding from external sources (Bolivia 2\% and Honduras 5\%) they do receive support from GAVI, the Vaccine Alliance for immunisation programmes, in common with a number of other LrMICs. ${ }^{49}$

Low-income nations are defined as those with GNI/ capita below US $\$ 1025$ per annum (in 2018), reflected in low (or unreported) levels of female secondary school enrolment, low rates of measles second-dose vaccines and high dependence on external sources for health expenditure. Many of these nations are in conflict zones or are recent victims of natural disaster. Limited health budgets allow little for public health measures, such as HPV immunisation programmes. Among LICs, Rwanda and Uganda report high HPV immunisation rates for 2018 ( $84 \%$ and $72 \%$, respectively). Both are close to the median for GNI/capita and health expenditure per capita but receive high proportions of health spending from external sources. Specifically, both are GAVI supported nations, with funded HPV immunisation programmes. ${ }^{49}$ Both countries have contributed to important lessons in how to structure such programmes, and particularly how to include hard-to-reach out-of-school girls. ${ }^{26} 5051$

The global HPV vaccination coverage rate in 2018 is calculated at $12.2 \%$. This is an approximation, based on only 1 year of data, combining different indicators as reported by individual countries. In those countries where vaccination registries are absent, these indicators are often survey based; where countries rely on opportunistic approaches to HPV vaccination, data are estimated. Where indicators are not reported, especially among richer nations, the assumption of no coverage likely underestimates some level of opportunistic vaccination. The estimated global coverage rate depends on a 


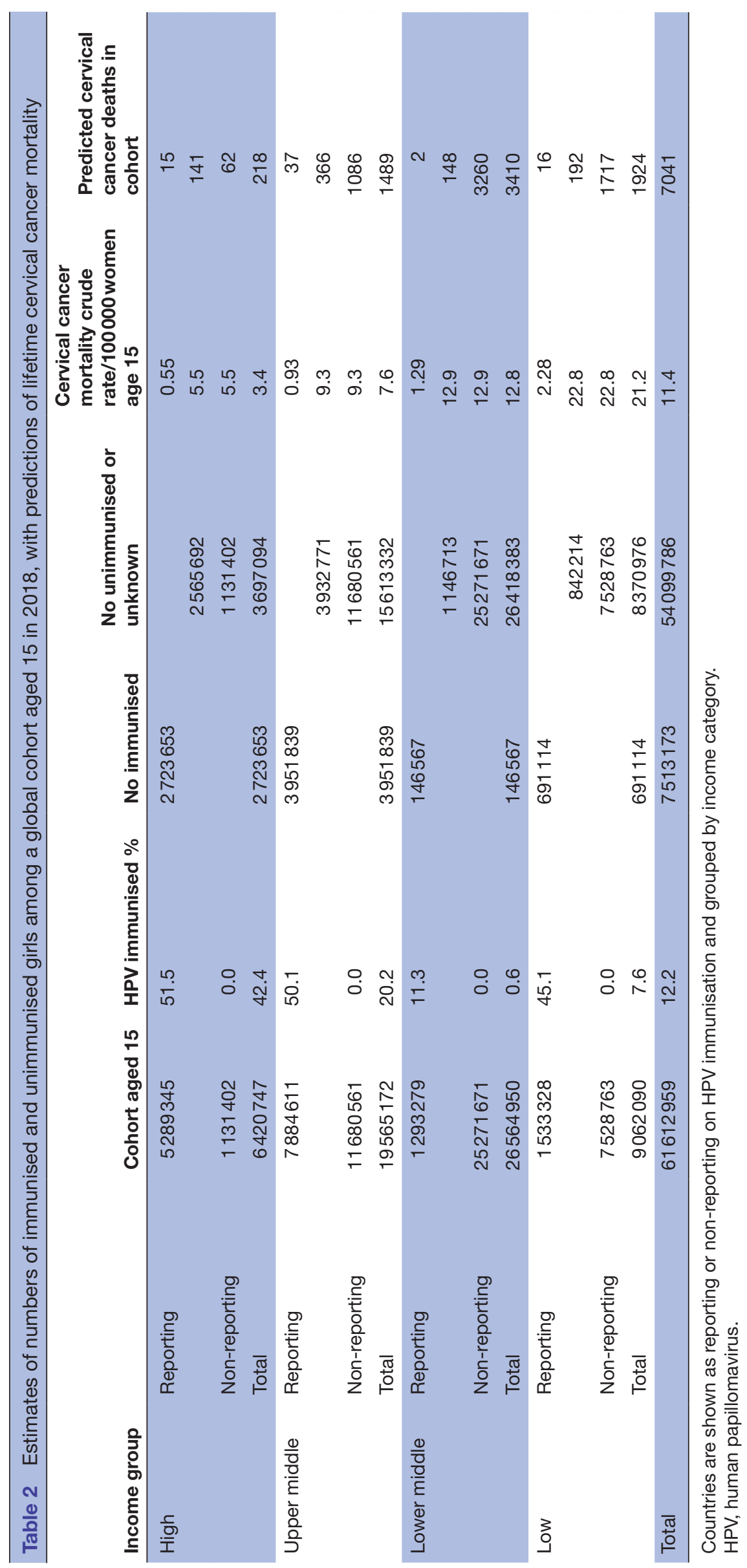


calculation of cohort size derived from a single year's data for childhood populations in each country.

Despite the limitations of the available data, the estimate of lifetime cervical cancer mortality in a single annual cohort of 15-year-old girls, demonstrates the wide gap between rich nations and all others. In HICs with a collective immunisation coverage rate of only $42 \%$, cervical cancer mortality for this cohort is forecast at 3.4 per 100000 women. Assuming secondary prevention and treatment approaches remain unchanged from the present, girls in this cohort in UMICs can expect a mortality rate more than twice as high as in HICs; in LrMICs almost four times as high and in LICs more than six times the HIC rate. This calculation assumes that mortality rates in the unimmunised population reflect current mortality rates. This is reasonable as the earliest vaccinated cohorts are only beginning to reach the age at which cervical cancer presents, and therefore, will not yet have reduced observed mortality rates. The calculation assumes HPV vaccination provides protection against $90 \%$ of cancers while some of the cohort will have received a vaccine offering $70 \%$ protection. On the other hand, partial protection provided by incomplete vaccine courses has been ignored. It is important to note that in HICs, with effective secondary prevention and treatment for cervical cancer, widespread HPV vaccination will save few lives. The primary benefits of immunisation in these settings will be to reduce reliance on invasive screening programmes and to reduce the expense and morbidity of the disease and its treatment.

These data strongly support the proposition that the burden of cervical cancer will continue to be borne by women in poor nations. However, there is cause for optimism. Eradicating cervical cancer is a recent priority initiative of WHO. ${ }^{32}$ Strategies to support this include ongoing updates to the database recording country-level HPV immunisation coverage. It now includes 107 countries recording vaccine course completion, with evidence of recent programme initiation in others. A July 2020 WHO report ${ }^{31}$ and follow-up study ${ }^{52}$ estimate global HPV immunisation coverage of $15 \%$, slightly above the rate estimated in this study, due mainly to recent introductions of immunisation programmes. A recent agreement with manufacturers is expected to increase supply of vaccine allowing GAVI to reach a broader population of girls in LMICs. ${ }^{53}$

Countries at all income levels need to maintain high uptake of HPV immunisation. Organised, fully funded, school-based programmes, monitored through centralised registries, offer the most effective strategy. These must be supported by information campaigns to increase awareness of cervical cancer and HPV immunisation, to address vaccine hesitancy and to reach out-ofschool girls.

\section{CONCLUSION}

Only the richest nations can currently afford routine HPV immunisation. For all others, even though cost-effectiveness is clear, healthcare budgets and competing priorities limit affordability. Success requires a commitment to universal health coverage and to secondary education for girls, as well as political will and strong leadership. Most importantly, initiatives to address affordability across all LMICs are required. Increased development assistance directed at HPV immunisation, as promised through multilateral organisations with cooperation from vaccine suppliers, will help financially and with technical support. A focus needs to be maintained on these objectives, despite global disruption and competing demands due to the COVID-19 pandemic.

Contributors JS: conceptualisation, data curation, verification of underlying data, data analysis, methodology, visualisation, writing-original draft, review and editing. TH: conceptualisation, verification of underlying data, methodology, writing-review and editing.

Funding The authors have not declared a specific grant for this research from any funding agency in the public, commercial or not-for-profit sectors.

Competing interests None declared.

Patient consent for publication Not required.

Ethics approval This research was based entirely on analysis of publicly accessible databases. No human or animal participants were involved and therefor no ethics approval was sought.

Provenance and peer review Not commissioned; externally peer reviewed.

Data availability statement All data relevant to the study are included in the article or uploaded as online supplemental information. No additional data available beyond that included in Supplementary Files.

Supplemental material This content has been supplied by the author(s). It has not been vetted by BMJ Publishing Group Limited (BMJ) and may not have been peer-reviewed. Any opinions or recommendations discussed are solely those of the author(s) and are not endorsed by BMJ. BMJ disclaims all liability and responsibility arising from any reliance placed on the content. Where the content includes any translated material, BMJ does not warrant the accuracy and reliability of the translations (including but not limited to local regulations, clinical guidelines, terminology, drug names and drug dosages), and is not responsible for any error and/or omissions arising from translation and adaptation or otherwise.

Open access This is an open access article distributed in accordance with the Creative Commons Attribution Non Commercial (CC BY-NC 4.0) license, which permits others to distribute, remix, adapt, build upon this work non-commercially, and license their derivative works on different terms, provided the original work is properly cited, appropriate credit is given, any changes made indicated, and the use is non-commercial. See: http://creativecommons.org/licenses/by-nc/4.0/.

ORCID iD

Jacqueline Spayne http://orcid.org/0000-0002-5128-2402

\section{REFERENCES}

1 Bray F, Ferlay J, Soerjomataram I, et al. Global cancer statistics 2018: GLOBOCAN estimates of incidence and mortality worldwide for 36 cancers in 185 countries. CA Cancer J Clin 2018;68:394-424.

2 Bosch FX, Lorincz A, Muñoz N, et al. The causal relation between human papillomavirus and cervical cancer. J Clin Pathol 2002;55:244-65.

3 Ferlay J, Ervik M, Lam F. Global cancer Observatory: cancer today: international agency for research on cancer, 2018. Available: https:// gco.iarc.fr/

4 Jansen EEL, Zielonke N, Gini A, et al. Effect of organised cervical cancer screening on cervical cancer mortality in Europe: a systematic review. Eur J Cancer 2020;127:207-23.

5 Koh Wui-Jin, Anderson BO, Carlson RW. NCCN resource-stratified and harmonized guidelines: a paradigm for optimizing global cancer care. Cancer 2020;126:2416-23.

6 Cohen PA, Jhingran A, Oaknin A, et al. Cervical cancer. The Lancet 2019;393:169-82. 
7 Jedy-Agba E, Joko WY, Liu B, et al. Trends in cervical cancer incidence in sub-Saharan Africa. Br J Cancer 2020;123:148-54

8 Sengayi-Muchengeti M, Joko-Fru WY, Miranda-Filho A, et al. Cervical cancer survival in sub-Saharan Africa by age, stage at diagnosis and Human Development Index: A population-based registry study. Int $J$ Cancer 2020;147:3037-48.

9 Chuang LT, Temin S, Camacho R, et al. Management and care of women with invasive cervical cancer: American Society of clinical oncology resource-stratified clinical practice guideline. Journal of Global Oncology 2016;2:311-40.

10 Papanicolaou GN, Traut HF. The diagnostic value of vaginal smears in carcinoma of the uterus. American Journal of Obstetrics and Gynecology 1941;42:193-206.

11 Catarino R, Schäfer S, Vassilakos P, et al. Accuracy of combinations of visual inspection using acetic acid or lugol iodine to detect cervical precancer: a meta-analysis. BJOG 2018;125:545-53.

12 Yeh PT, Kennedy CE, de Vuyst H, et al. Self-Sampling for human papillomavirus (HPV) testing: a systematic review and meta-analysis. BMJ Glob Health 2019;4:e001351.

13 La Torre G, de Waure C, Chiaradia G, et al. Hpv vaccine efficacy in preventing persistent cervical HPV infection: a systematic review and meta-analysis. Vaccine 2007:25:8352-8.

14 Lu B, Kumar A, Castellsagué X, et al. Efficacy and safety of prophylactic vaccines against cervical HPV infection and diseases among women: a systematic review \& meta-analysis. BMC Infect Dis 2011;11:13

15 Lehtinen M, Dillner J. Clinical trials of human papillomavirus vaccines and beyond. Nat Rev Clin Oncol 2013;10:400-10.

16 Lowy DR, Schiller JT. Reducing HPV-associated cancer globally. Cancer Prev Res 2012;5:18-23.

17 Drolet M, Bénard Élodie, Boily M-C, et al. Population-Level impact and herd effects following human papillomavirus vaccination programmes: a systematic review and meta-analysis. Lancet Infect Dis 2015;15:565-80.

18 Drolet M, Bénard Élodie, Pérez N, et al. Population-level impact and herd effects following the introduction of human papillomavirus vaccination programmes: updated systematic review and metaanalysis. The Lancet 2019;394:497-509.

19 Bonanni P, Levi M, Latham NB, et al. An overview on the implementation of HPV vaccination in Europe. Hum Vaccin 2011;7:128-35.

20 Gervais F, Dunton K, Jiang Y, et al. Systematic review of costeffectiveness analyses for combinations of prevention strategies against human papillomavirus (HPV) infection: a general trend. BMC Public Health 2017;17:283.

21 Paul P, Fabio A. Literature review of HPV vaccine delivery strategies: considerations for school- and non-school based immunization program. Vaccine 2014;32:320-6.

22 Arrossi S, Temin S, Garland S, et al. Primary prevention of cervical cancer: American Society of clinical oncology resource-stratified guideline. J Glob Oncol 2017;3:611-34.

23 Bello FA, Enabor OO, Adewole IF. Human papilloma virus vaccination for control of cervical cancer: a challenge for developing countries. Afr J Reprod Health 2011;15:25-30.

24 Bruni L, Diaz M, Barrionuevo-Rosas L, et al. Global estimates of human papillomavirus vaccination coverage by region and income level: a pooled analysis. Lancet Glob Health 2016;4:e453-63.

25 Loke AY, Kwan ML, Wong Y-T, et al. The uptake of human papillomavirus vaccination and its associated factors among adolescents: a systematic review. J Prim Care Community Health 2017:8:349-62.

26 Gallagher KE, Howard N, Kabakama S, et al. Human papillomavirus (HPV) vaccine coverage achievements in low and middle-income countries 2007-2016. Papillomavirus Res 2017;4:72-8.

27 Gallagher KE, LaMontagne DS, Watson-Jones D. Status of HPV vaccine introduction and barriers to country uptake. Vaccine 2018;36:4761-7.

28 Altobelli E, Rapacchietta L, Profeta VF, et al. HPV-vaccination and cancer cervical screening in 53 who European countries: an update on prevention programs according to income level. Cancer Med 2019:8:2524-34.

29 Nguyen-Huu N-H, Thilly N, Derrough T, et al. Human papillomavirus vaccination coverage, policies, and practical implementation across Europe. Vaccine 2020;38:1315-31.
30 WHO/UNICEF. Progress and challenges with achieving universal immunization coverage 2019, 2020. Available: https://www.who.int/ docs/default-source/documents/immunization/data/who-unicefimmunisation.pdf?sfvrsn $=6 \mathrm{bc} 33 \mathrm{c} 2 \mathrm{~d} \_2$

$31 \mathrm{WHO} / \mathrm{UNICEF}$. Progress and challenges with achieving universal immunization coverage 2020, 2020. Available: https://www.who.int/ immunization/monitoring surveillance/who-immuniz.pdf

32 WHO. Draft global strategy towards the elimination of cervical cancer as a public health problem. Geneva: World Health Organization, 2019. https://www.who.int/publications/m/item/draft-global-strategytowards-eliminating-cervical-cancer-as-a-public-health-problem

33 World_Bank. World bank country and lending groups 2020, 2020. Available: https://datahelpdesk.worldbank.org/knowledgebase/ articles/906519-world-bank-country-and-lending-groups

34 IARC. Global cancer observatory Lyon. France: International Agency for Research on Cancer, 2020. https://gco.iarc.fr/

35 Ferlay J, Colombet M, Soerjomataram I, et al. Estimating the global cancer incidence and mortality in 2018: GLOBOCAN sources and methods. Int J Cancer 2019;144:1941-53.

36 World Bank. World Bank open data 2020, 2020. Available: https:// data.worldbank.org/

37 World Bank. World development indicators Washington DC, USA 2020. Available: https://databank.worldbank.org/source/worlddevelopment-indicators

38 Unicef. Sample of the WHO/UNICEF joint report form on immunization New York, USA: UNICEF, 2020. Available: https://data. unicef.org/topic/child-health/immunization/

39 Unicef. Immunization data 2020. available from. Available: https:// data.unicef.org/topic/child-health/immunization/ [Accessed 15 March 2020].

40 Unicef. WHO estimates of human papillomavirus immunization coverage 2010-2018 - database UNICEF, 2019. Available: https:// data.unicef.org/resources/dataset/immunization/

41 Huh WK, Joura EA, Giuliano AR, et al. Final efficacy, immunogenicity, and safety analyses of a nine-valent human papillomavirus vaccine in women aged $16-26$ years: a randomised, double-blind trial. The Lancet 2017:390:2143-59.

42 IARC. DATA \& METHODS 2020, 2020. Available: https://gco.iarc.fr/ today/data-sources-methods

43 Buscail C, Gagnière B. Vaccination coverage of adolescents: results of a defense and citizenship Day-based survey. Médecine et Maladies Infectieuses 2016;46:25-31.

44 Yamaguchi M, Sekine M, Kudo R, et al. Differential misclassification between self-reported status and official HPV vaccination records in Japan: implications for evaluating vaccine safety and effectiveness. Papillomavirus Res 2018;6:6-10.

45 Borena W, Luckner-Hornischer A, Katzgraber F, et al. Factors affecting HPV vaccine acceptance in West Austria: do we need to revise the current immunization scheme? Papillomavirus Res 2016:2:173-7.

46 Wymann MN, Zographos AS, Altpeter E, et al. Human papillomavirus vaccine uptake in adolescence and adherence to cervical cancer screening in Switzerland: a national cross-sectional survey. Int J Public Health 2018;63:105-14.

47 Hansen PR, Schmidtblaicher M, Brewer NT. Resilience of HPV vaccine uptake in Denmark: decline and recovery. Vaccine 2020;38:1842-8.

48 Cernuschi T, Gilchrist S, Hajizada A, et al. Price transparency is a step towards sustainable access in middle income countries. $B M J$ 2020;368:15375.

49 GAVI. Country information, 2020. Available: https://www.gavi.org/ programmes-impact/country-hub/africa

50 Bonner K, Banura C, Basta NE. Hpv vaccination strategies targeting hard-to-reach populations: out-of-school girls in LMICs. Vaccine 2018;36:191-3.

51 Mukakalisa I, Bindler R, Allen C, et al. Cervical cancer in developing countries: effective screening and preventive strategies with an application in Rwanda. Health Care Women Int 2014;35:1065-80.

52 Bruni L, Saura-Lázaro A, Montoliu A, et al. HPV vaccination introduction worldwide and who and UNICEF estimates of national HPV immunization coverage 2010-2019. Prev Med 2021:144:106399.

53 Simelela N. Cervical cancer elimination initiative updates: WHO, 2020. Available: https://www.who.int/initiatives/cervical-cancerelimination-initiative 